\title{
Editorial: Electronic Commerce in the Time of Covid-19 - Perspectives and Challenges
}

\author{
Mirjana Pejić-Bach ${ }^{1}$ \\ ${ }^{1}$ University of Zagreb, Faculty of Economics \& Business, Department of Informatics, Zagreb, Croatia, mpejic@efzg.hr \\ Co-Editor \\ January 2021
}

The Covid-19 epidemic has strongly affected the lives and economies of the countries all over the world, generating numerous challenges for private, public, and business entities. Massive quarantines have been implemented globally that stopped most of the interactions between people, which is popularly referred to as social distancing. However, social distancing implies the minimization of physical contact among humans. At the same time, people stayed virtually connected due to the abundance of various electronic commerce solutions. The Covid-19 epidemic has rapidly forced numerous entities (public, business, and private) to become predominantly oriented to electronic commerce in the last few months. It is not too exaggerated to say that electronic commerce has enabled the survival of numerous businesses all over the world, and has supported various personal needs, as well as public functions. Suddenly, new perspectives and challenges emerge due to the usage of electronic commerce processes and practices in the time of the Covid-19 crises.

The examples of such perspectives and challenges are abundant. However, the pattern emerges, and in most cases, electronic commerce technologies are initially perceived as savior solutions, various challenges emerge in the middle term, while in the long term the realistic attitude towards electronic commerce technology should be established.

One of the most striking examples of the utilization of electronic commerce in the time of the Covid-19 crisis is communication. In the time of the Covid-19 quarantine, electronic commerce remains the sole source of various types of communication addressing both business and personal needs. Quarantine forces most of us to communicate in person solely to the members of our households. Video services, such as Skype, Zoom, and Google Meet has become the primary way for communication with people outside of our households - family members, friends, teachers, students, business partners, co-workers, and clients. In brief, without video conferencing solutions, face-to-face communication would not be possible with people outside of our households. Therefore, in the short term, video conferencing services are perceived as saviors for various processes, such as collaborating with colleagues in the office and delivering e-learning solutions. However, in the middle term, the problems with the usage of video services emerged rapidly, mostly from the lack of security and privacy, curbing at least to some extent the initial enthusiasm and frustrations emerge. Besides, the dissatisfaction with video conferencing is rising, due to the bitter truth that such type of communication cannot replace in-person communication, due to various reasons. To name just one, video conferencing disables the communication of various non-verbal clues, which are enormously relevant in our relationships. At the same time, the long-term perspective emerges. For example, it has become evident that video conferencing is much more useful in case of the meetings of geographically distant people than was previously perceived. Therefore, it can be expected that video conferencing will continue to be used after the Covid-19 epidemic ends since it will significantly decrease the costs of such meetings, which will have in turn huge positive feedback on the environment.

A similar discussion could be developed for various examples of electronic commerce that have become widely used in the time of the Covid-19 epidemic, such as business practices (e.g. capacity for the distribution of goods and services has become the bottleneck of the supply chain management), privacy and security (e.g. sharing various content using ad-hoc electronic commerce solutions without using the proper privacy shields), and mobile connectivity (e.g. although people stay at home for social distancing, they become available non-stop for he work-related communication).

In light of our current experience, a wide area of scientific research should be developed. The goal of such research should be to develop both theoretical and practical contributions. One of the possible theoretical contributions lies in the field of the various theories of technology acceptance, which probably should be redesigned, taking into account that people accept technology differently in the time of crisis. Practical contributions should encompass various aspects of the short term, middle term, and long-term usage of electronic solutions in the time of the Covid-19 crises, such as communication.

Finally, we should take into account that the Covid-19 epidemics will eventually stop in the future, leaving us with the scarves that resulted from the disease itself and the consequences of the quarantine. However, all of this would be in vain if we all do not work strongly in the future to retain the examples of good practices, to improve them, and to solve the problems that surfaced, such as various privacy and security issues. All of the current knowledge and experiences should be transferred into valid, scientifically based research, as soon as possible, that would provide a solid basis for understanding how electronic commerce can support the 'new normality' that we are facing in the period ahead of us. 